\title{
El método gráfico y analítico en la teoría de mecanismos
}

\section{The graphical and analytical method in the theory of mechanisms}

\author{
VÁZQUEZ-GONZÁLEZ, Benjamín†*, JIMÉNEZ-RABIELA, Homero, RAMÍREZ-CRUZ, José Luis \\ y QUINTANA-DIAZ, María Berenice Guadalupe
} Universidad Autónoma Metropolitana, Unidad Azcapotzalco, División de Ciencias Básicas e Ingeniería, Departamento de
Energía

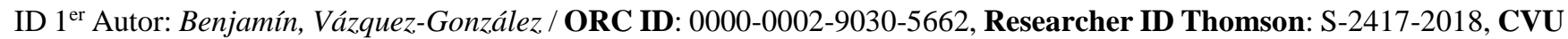
CONACYT ID: 25749

ID $1^{\text {er }}$ Coautor: Homero, Jiménez-Rabiela / Researcher ID Thomson: S-2299-2018, CVU CONACYT ID: 123386

ID $2^{\text {do }}$ Coautor: José Luis, Ramírez-Cruz / ORC ID: 0000-0003-0762-2630, Researcher ID Thomson: G-3405-2019, CVU CONACYT ID: 921268

ID $3^{\text {er }}$ Coautor: María Berenice Guadalupe, Quintana-Diaz / ORC ID: 0000-0002-4703-1934, CVU CONACYT ID: 1015523

DOI: $10.35429 / J T D .2019 .10 .3 .24 .29$

Recibido: 10 de Marzo, 2019; Aceptado 30 de Junio, 2019

\begin{abstract}
Resumen
La teoría de mecanismos es una de las ramas fundamentales de estudio y desarrollo en la teoría de máquinas, los actuales desarrollos tecnológicos tales como la mecatrónica o la robótica requieren que los ingenieros e investigadores posean conocimientos básicos sólidos en mecánica aplicada, esto permitirá realizar investigación confiable y eficiente. La enseñanza tradicional en las carreras de ingeniería emplea bibliografía que presenta de manera aislada, ya sea el método gráfico o bien el analítico, sin establecer una clara correlación entre ambas metodologías, con lo que se pierde la oportunidad de que dichas metodologías se sustenten mutuamente. Cuando dos metodologías se complementan se obtiene un nivel de seguridad en el dominio de las técnicas de solución de problemas, a su vez, se abre la posibilidad de extender soluciones cuando se busca la representación de una de las metodologías, basada en la otra. En este trabajo se presenta la solución de problemas de la teoría de mecanismos por ambas metodologías, explicando y justificado los resultados obtenidos, así como la secuencia de pasos en la solución de problemas, más allá de un procedimiento.
\end{abstract}

Educación, Mecanismos, Cinemática

\begin{abstract}
The theory of mechanisms is one of the fundamental branches of study and development in the theory of machinery, current technological developments such as mechatronics or robotics require that engineers and researchers have solid basic knowledge in applied mechanics; this will allow reliable and efficient research. The traditional teaching in the engineering fields uses bibliography that presents in an isolated way, either the graphic or the analytical method, without establishing a clear correlation between both methodologies, which misses the opportunity for these methodologies to mutually support each other. When two methodologies complement each other, a level of security is obtained in the domain of problem solving techniques, in turn, opens the possibility of extending solutions when looking for the representation of one of the methodologies, based on the other, in this paper presents the solution of problems of the theory of mechanisms by both methodologies, explaining and justifying the results obtained, as well as the sequence of steps in the solution of problems, beyond a procedure.
\end{abstract}

Education, Mechanisms, Kinematics

Citación: VÁZQUEZ-GONZÁLEZ, Benjamín, JIMÉNEZ-RABIELA, Homero, RAMÍREZ-CRUZ, José Luis y QUINTANA-DIAZ, María Berenice Guadalupe. El método gráfico y analítico en la teoría de mecanismos. Revista del Desarrollo Tecnológico. 2019. 3-10: 24-29

\footnotetext{
* Correspondencia del Autor (Correo electrónico: bvg@correo.azc.uam.mx)

$\dagger$ Investigador contribuyendo como primer autor.
} 


\section{Introducción}

En este trabajo se desarrolla el tema del análisis cinemático de mecanismos en una forma más general a la convencional, es conocido el avance que se tiene desde el estudio de los mecanismos hasta la robótica actualmente ver, Staicu y Vu. En literatura tradicional el análisis cinemático de mecanismos, dependiendo de la bibliografía, se presenta el método gráfico y un método analítico, Shigley aborda de manera preferencial el método gráfico para la solución cinemática del problema de la velocidad, por otro lado, también desarrolla soluciones por medio del método analítico, sin embargo, no establece una correlación directa entre ambos métodos.

Norton por su parte, privilegia el método analítico en su versión del uso de la variable compleja, sobre el gráfico, lo cual limita el aprovechamiento cognitivo en el aprendizaje, no establece una correlación directa entre ambas metodologías. Otros autores como por ejemplo Beer y Jhonston, que también estudian estos temas, se abocan a soluciones analíticas vectoriales sin construir una representación gráfica, como lo es un polígono de velocidades, por lo que tampoco se logran vincular ambas metodologías. El problema a tratar en este trabajo es el de vincular ambas metodologías, la construcción vectorial de un polígono de velocidades y la solución analítica.

El método gráfico cómo se presenta en la literatura, consta de una serie de operaciones gráficas, resultado de las características cinemáticas desarrolladas por los eslabones de un mecanismo. Esta forma de solución gráfica produce que se recurra a un procedimiento que sistematiza las operaciones de manera visual, dedo que el método es gráfico. Esta es una limitación de dicho método. Sin embargo, tener una concepción completa de las operaciones realizadas y su significado cinemático, es una gran aportación del método gráfico si se acompaña de una explicación analítica.

En la siguiente sección se desarrolla en primer lugar el análisis gráfico de un mecanismo de cuatro eslabones, se utilizan los procedimientos asimilados en el aprendizaje de las referencias mencionadas con anterioridad. En la sección tres, se desarrollan las expresiones vectoriales correspondientes, para obtener las soluciones analíticas equivalentes.
En la sección cuatro se vinculan las soluciones determinadas en cada caso. El procedimiento empleado es a través de un ejemplo numérico, lo cual aporta claridad y es una metodología muy didáctica en los temas de enseñanza en el campo de la ingeniería en la rama de mecanismos.

\section{Análisis de la velocidad de un mecanismo de cuatro eslabones por el método gráfico}

Se inicia el análisis cinemático con el desarrollo del método gráfico, para ilustrar los procedimientos tradicionales. En la Figura 1 se muestra un eslabonamiento de cuatro barras.

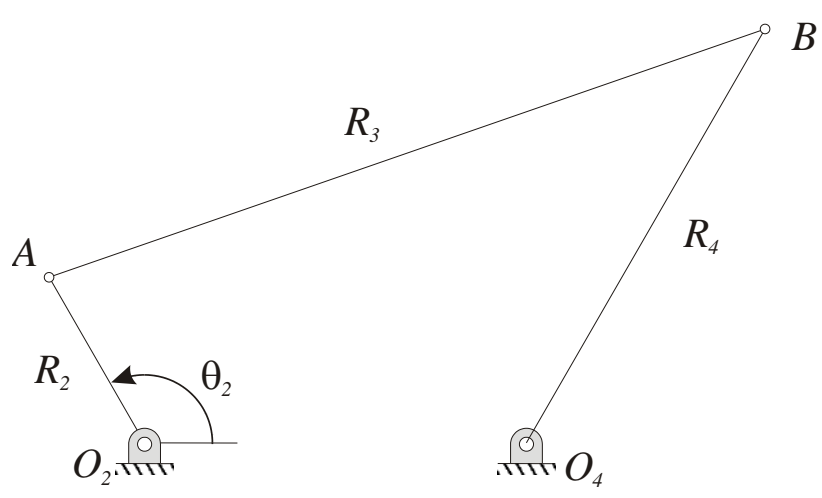

Figura 1 Mecanismo típico de cuatros eslabones en una configuración abierta

Los valores de los parámetros del mecanismo se presentan en la Tabla 1, hay que recordar que a los eslabones se les puede asociar características vectoriales, por tener magnitud, dirección y sentido, en la tabla 1 se registran las magnitudes correspondientes.

\begin{tabular}{|l|l|}
\hline $\mathrm{R}_{1}=0.04 \mathrm{~m}$ & $\mathrm{R}_{2}=0.02 \mathrm{~m}$ \\
\hline $\mathrm{R}_{3}=0.0794 \mathrm{~m}$ & $\mathrm{R}_{4}=0.05 \mathrm{~m}$ \\
\hline$\theta_{2}=120^{\circ}$ & $\omega_{2}=+60 \mathrm{rad} / \mathrm{s}$ \\
\hline
\end{tabular}

Tabla 1 Datos numéricos del mecanismo de la Figura 1

El análisis de la velocidad por medio del método gráfico, emplea la expresión de Euler,

$V_{A}=V_{O 2}+\omega_{2} \times R_{2}$

Donde $\boldsymbol{V}_{\boldsymbol{O} 2}$ es cero por permanecer fijo. La velocidad del punto $A$ se localiza desarrollando gráficamente el producto vectorial entre $\boldsymbol{\omega}_{2}$ y $\boldsymbol{R}_{2}$, esto resulta en un vector perpendicular a $R_{2}$, y con magnitud igual a,

$V_{A}=\omega_{2} R_{2}=(60)(0.02)=1.2 \mathrm{~m} / \mathrm{s}$ 
El siguiente paso es definir arbitrariamente una escala para representar el vector velocidad instantánea del punto $A$. Se define la siguiente escala: $2 \mathrm{~cm} \rightarrow 1.2 \mathrm{~m} / \mathrm{s}$, de esta forma se tienen todos los datos para localizar en un sistema coordenado, al vector velocidad del punto $A$. Este es un vector que tiene una magnitud de $5 \mathrm{~cm}$ y una dirección perpendicular al eslabón $R_{2}$. La representación gráfica de lo anterior se denomina paso 1 ).

El siguiente paso es determinar la velocidad del punto $B$, a partir del punto $O_{4}$. Esto se logra aplicando nuevamente la expresión de Euler, en estos nuevos términos se tiene,

$V_{B}=V_{O 4}+\omega_{4} \times R_{4}$

Donde $V_{O 4}$ es cero por permanecer fijo. Se observa que la incógnita en esta expresión es el vector $\omega_{4}$, la cual se determinará gráficamente. Sin embargo se puede representar la información contenida en la ecuación (2). Esta información parcial corresponde con la dirección del vector velocidad del punto $B$, la cual se determina nuevamente realizando el producto vectorial entre $\omega_{4} \mathrm{y} R_{4}$, esto resulta en una línea recta, es decir representa la dirección del vector velocidad del punto $B$, pero no es un vector porque se desconoce su sentido y su magnitud. Entonces el siguiente paso es trazar una línea recta que parte del punto $B$ con una magnitud indefinida. Este paso gráfico de denomina paso 2 ).

Los pasos anteriores se ilustraran en la figura final, que contiene al polígono de velocidades completo. Sin embargo, todavía no se presenta dicho polígono, porque todavía se debe realizar un paso más. La tercera etapa o paso 3), corresponde con el cálculo de la velocidad del punto $B$, desde el punto $A$. Esto también se realiza con la expresión de Euler aplicada entre dichos puntos, lo anterior se escribe de la siguiente manera,

$V_{B}=V_{A}+\omega_{3} \times R_{3}$

En el trazo de los polígonos de velocidad, lo anterior implica que a partir del vector conocido $V_{A}$, se suma vectorialmente el producto $\omega_{3} \times R_{3}$. Finalmente este producto vectorial no se puede evaluar completamente, porque se desconoce la magnitud del vector $\omega_{3}$, dado que esta es una incógnita.
Sin embargo, es posible determinar la dirección que tiene ese vector, dicha dirección es perpendicular al vector $R_{3}$, por lo que en el polígono de velocidades, se trazará una línea recta a partir del vector $V_{A}$. Esto último resulta en el denominado polígono de velocidades, que como su nombre lo indica es un objeto geométrico cerrado por las intersecciones de la rectas determinadas con anterioridad. Al obtener las intersecciones, se pueden determinar las magnitudes de los vectores del polígono de velocidades. En la Figura 2 se muestra el mecanismo original y su correspondiente polígono de velocidades.

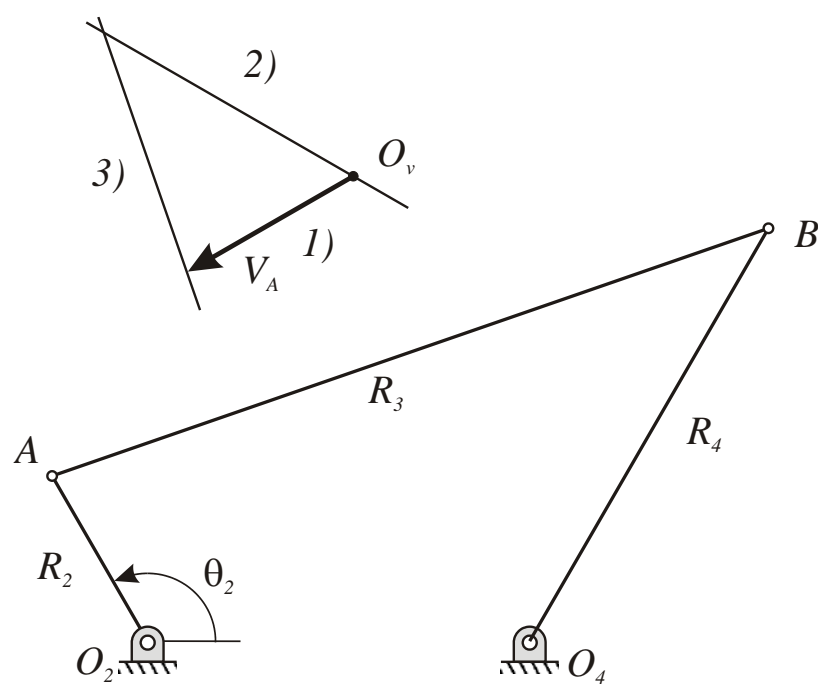

Figura 2 Mecanismo de cuatro eslabones junto con su polígono de velocidades

Hay que notar que la intersección entre las líneas 2) y 3) definen las magnitudes de los vectores correspondientes a las velocidades siguientes, $V_{B}$ y $V_{B A}$.

En la Figura 3 se muestra el polígono de velocidades completo donde se observa también, las direcciones de los vectores que se han determinado.

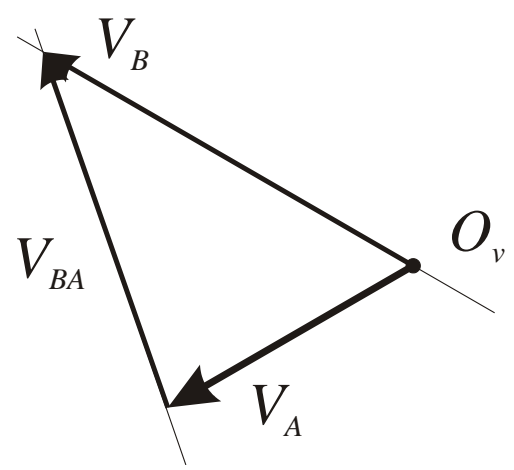

Figura 3 Polígono de velocidades construido a partir de la metodología gráfica tradicional 
En la Tabla 2 se registran las longitudes de las líneas que se han intersectado y conforme a la escala que se designó originalmente, se determinan los valores de velocidad de los vectores correspondientes.

\begin{tabular}{|l|l|}
\hline$V_{B} \rightarrow 3.02 \mathrm{~cm}$ & $V_{B}=1.812 \mathrm{~m} / \mathrm{s}$ \\
\hline$V_{B A} \rightarrow 2.66 \mathrm{~cm}$ & $V_{B A}=1.596 \mathrm{~m} / \mathrm{s}$ \\
\hline
\end{tabular}

Tabla 2 Valores de las velocidades de desplazamiento instantáneas obtenidas por medio del método gráfico

El último paso en el análisis de velocidades por medio del método gráfico, corresponde con determinar las velocidades angulares, esto se realiza a continuación.

De ecuación 2 se considera la magnitud del producto vectorial, como dichos vectores son perpendiculares entre sí, el resultado es,

$V_{B}=\omega_{4} R_{4}$

de donde se obtiene,

$\omega_{4}=\frac{V_{B}}{R_{4}}=\frac{1.812 \mathrm{~m} / \mathrm{s}}{0.05 \mathrm{~m}}=36.24 \frac{\mathrm{rad}}{\mathrm{s}}$

De la ecuación (3) se obtiene lo siguiente

$V_{B A}=V_{B}-V_{A}=\omega_{3} \times R_{3}$

Tomando en cuenta la perpendicularidad entre el vector velocidad angular y el eslabón $\boldsymbol{R}_{3}$, resulta

$\omega_{3}=\frac{V_{B A}}{R_{3}}=\frac{1.596 \mathrm{~m} / \mathrm{s}}{0.0794 \mathrm{~m}}=20.1 \frac{\mathrm{rad}}{\mathrm{s}}$

Análisis de la velocidad de un mecanismo de cuatro eslabones por el método analítico

En esta sección se obtiene la solución del análisis de velocidad por medio del método analítico.

El método analítico se puede desarrollar por medio de la variable compleja o por análisis vectorial, ambas estrategias proporcionan los mismos resultados. Aquí se empelaran las ecuaciones, resultado de utilizar la variable compleja. La solución del problema de la cinemática en mecanismos y en particular de la velocidad se inicia por el planteamiento de la ecuación de cierre de circuito. El objetivo de este trabajo es correlacionar directamente el método gráfico con el método analítico. A continuación se presentan las ecuaciones, resultado de aplicar el método analítico.
El método analítico requiere de haber realizado previamente el análisis de posición, esto permite determinar los valores de las posiciones angulares de los eslabones 2 y 3 respectivamente. Para esto se emplean las ecuaciones de Freundenstein, estas ecuaciones son muy conocidas, Norton las expone con claridad. Los resultados son los siguientes para el caso de estudio:

$\theta_{3}=19.082^{\circ}=0.33305 \mathrm{rad}, \mathrm{y} \theta_{4}=60^{\circ}=1.0472$ rad.

La ecuación de cierre de circuito o de circuito cerrado es,

$R_{2}+R_{3}=R_{1}+R_{4}$

Para el cálculo de la velocidad, se deriva en el tiempo la ecuación (6), y desarrollando ésta expresión en la forma polar compleja, con $\boldsymbol{R}=$ $R e^{\imath \theta}$, en cada vector respectivamente se obtiene una igualdad, que incluye términos reales y complejos. Ahora, separando la parte real y compleja, se obtiene el siguiente sistema de ecuaciones,

$$
\begin{aligned}
& \omega_{3} R_{3} \cos \theta_{3}-\omega_{4} R_{4} \cos \theta_{4}=-\omega_{2} R_{2} \cos \theta_{2} \\
& Y \\
& \omega_{3} R_{3} \operatorname{sen} \theta_{3}-\omega_{4} R_{4} \operatorname{sen} \theta_{4}=-\omega_{2} R_{2} \operatorname{sen} \theta_{2}
\end{aligned}
$$

Realizando el cambio de variables $x=\omega_{3} R_{3} \quad$ y $\quad y=\omega_{4} R_{4} \quad$ y junto con los datos numéricos del presente problema, las ecuaciones (7) y (8) se convierten en el siguiente sistema de ecuaciones algebraico,

$$
\begin{aligned}
& 0.94505 x-0.5 y=0.6 \\
& 0.32693 x-0.86603 y=-1.0392
\end{aligned}
$$

La solución numérica de este sistema tiene por resultados $x=1.5867$ y $y=1.799$, despejando de éstos valores numéricos se obtiene,

$$
\begin{aligned}
& \omega_{3}=\frac{x}{R_{3}}=\frac{1.5867}{0.0794}=19.984 \frac{\mathrm{rad}}{\mathrm{s}} \\
& \omega_{4}=\frac{y}{R_{4}}=\frac{1.799}{0.05}=35.98 \frac{\mathrm{rad}}{\mathrm{s}}
\end{aligned}
$$

Estos resultados son muy aproximados a los obtenidos en las ecuaciones (5) y (7), que provienen de una solución gráfica y por tanto aproximada. 
Obsérvese que la intersección de las curvas de la Figura 4, corresponde con la solución del sistema de ecuaciones, lo que corresponde con una representación gráfica de la solución analítica.

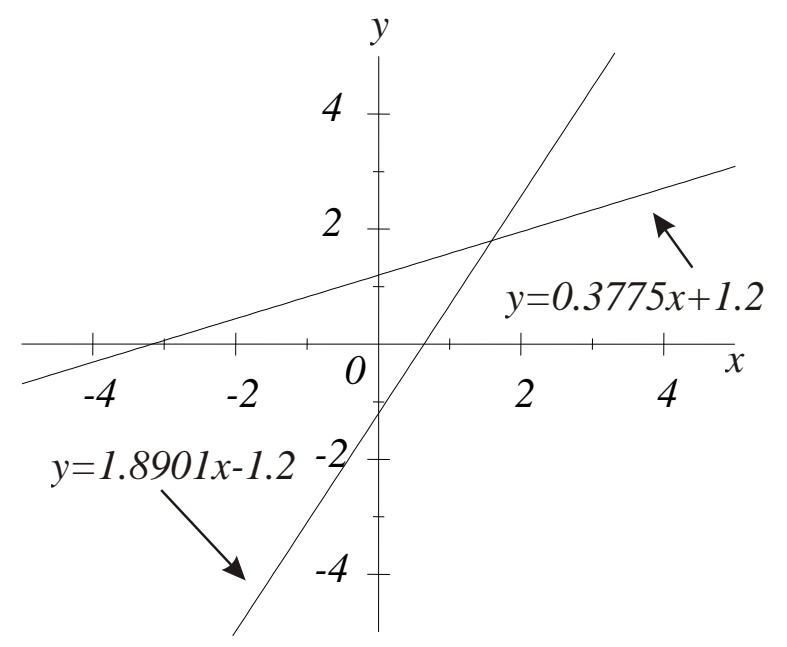

Figura 4 Solución gráfica del sistema de ecuaciones (10)

\section{Vinculación entre la solución gráfica y la analítica}

En esta sección se establece una relación directa y tangible de las soluciones por medio del polígono de velocidades y el método analítico.

En las secciones anteriores se desarrollaron de manera individual los dos métodos considerados. La vinculación que justifica los trazos que se deben realizar en el método gráfico en el sentido de que hay implícita una solución analítica, se proporciona a continuación.

En un sistema de ecuaciones como el de la ecuación (10), las constantes están en el espacio de la posición, esto se confirma porque las constantes coinciden con los ángulos de los eslabones. Las soluciones $x$ y $y$ encontradas, también están en el espacio de configuración de la posición, la rotación que se describe por el producto vectorial $\boldsymbol{\omega} \times \boldsymbol{R}$, lleva del espacio de configuración de la posición, al espacio de configuración de la velocidad, entonces es de esperarse que al rotar de manera ortogonal las soluciones gráficas, estas tengan una posición ortogonal con respecto a los eslabones, puesto que son parte de la solución.
Esto se observa en la Figura 5, al colocar las gráficas de las soluciones analíticas de la Figura 4, con ángulo de giro de $90^{\circ}, y_{1}$ corresponde con la rotación a $90^{\circ}$ de la ecuación $y=1.8901 x-1.2$ y $y_{2}$ corresponde con la rotación de la ecuación $y=0.3775 x+1.2$.

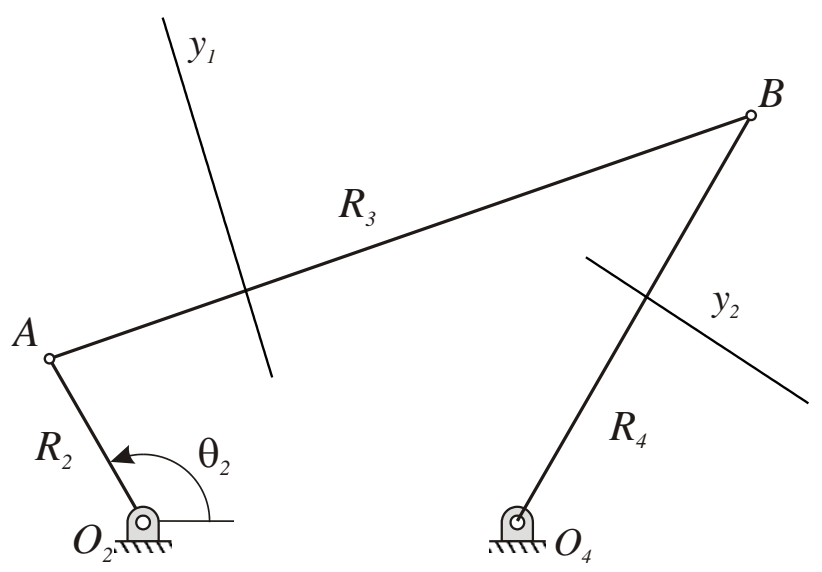

Figura 5 Mecanismo original con las soluciones $\mathrm{y}_{1}=1.8901 \mathrm{x}-1.2$ con rotación de $90^{\circ}, \mathrm{y} \mathrm{y}_{2}=0.3775 \mathrm{x}+1.2$ con rotación de $90^{\circ}$

Lo anterior justifica y complementa a ambos métodos, cuando se determina la velocidad por el método analítico se están hallando numéricamente los valores de la velocidad. Por otro lado, la solución gráfica se explica al hallar las intersecciones de los vectores perpendiculares a los eslabones.

\section{Agradecimiento}

Los autores agradecemos el apoyo institucional, de la Universidad Autónoma Metropolitana y de la Unidad Azcapotzalco, en particular de la División de Ciencias Básicas e Ingeniería y del Departamento de Energía.

\section{Conclusiones}

En este trabajo se ha determinado la correlación entre el método gráfico y el método analítico en el análisis cinemático de la velocidad de un mecanismo de cuatros eslabones. La descripción tradicional del método gráfico, aporta una serie de instrucciones a seguir en forma de trazos que en ocasiones pueden llegar a ser ambiguos en su interpretación, en el sentido de ser una mera instrucción, sin tener el sustento formal de obtener una solución analítica. Al correlacionar la solución analítica e interpretar su efecto en el contexto de la velocidad, se ha confirmado que la solución gráfica, implica muy cercanamente la solución analítica. 


\section{Referencias}

Beer, F. P. Johnston, E. R., Cornwell, P. J. (2013), Mecánica Vectorial para Ingenieros.

USA. McGraw-Hill

J. E. Shigley y J. J. Uicker. (1980). Theory of Machines and Mechanisms. USA: McGrawHill.

Norton R. L.. (2009). Kinematics and Dynamics of Machinery. USA: McGraw Hill Springer, Cham.

Staicu, S. (2019). Geared Parallel Mechanisms. In Dynamics of Parallel Robots (pp. 245-275).

Vu, L. N., \& Kuo, C. H. (2019). An analytical stiffness method for spring-articulated planar serial or quasi-serial manipulators under gravity and an arbitrary load. Mechanism and Machine Theory, 137, 108-126. 